\title{
Über das spontane Kristallisationsvermögen bei Wismut und Antimon.
}

\author{
Von \\ E. BEKIER.
}

Wie bekannt ${ }^{1}$ bestimmen 2 Hauptfaktoren, die lineare Kristal. lisationsgeschwindigkeit und das spontane Kristallisationsvermögen, den Kristallisationsprozeß. Bei Metallen können wir infolge ihrer Undurchsichtigkeit sowie der wahrscheinlich großen linearen Kristallisationsgeschwindigkeit wegen diese Faktoren im einzelnen nicht messen, wie es bei anderen Körpern ${ }^{2}$ möglich ist. Indem wir aber die innere Struktur der Metallreguli, die mit verschiedener Geschwindigkeit abgekühlt wurden, betrachten, können wir mittelbar dennoch über die Abhängigkeit des Verhältnisses:

$$
\text { lineare Kristallisationsgeschwindigkeit }
$$

von der Unterkühlung ein Urteil gewinnen.

Von diesem Verhältnis ausgehend können wir folgende Fälle von Kristallisationsprozessen unterscheiden: Ist das Verhältnis unabhängig von der Abkühlungsgeschwindigkeit, so darf man erwarten, daß die Größe der einzelnen Kristallindividuen von der Abkühlungsgeschwindigkeit unabhängig ist. Wächst jenes Verhältnis mit der Unterkühlung, so muß mit wachsender Abkühlungsgeschwindigkeit die Größe der einzelnen Kristallindividuen kleiner werden, nimmt aber jenes Verbältnis mit wachsender Unterkühlung ab, so werden sich die Kristallite des Konglomerates mit wachsender Abkühlungsgeschwindigkeit vergrößern.

Die mit Wismut und Antimon angestellten Versuche zeigen, daß die Größe der Kristallite mit wachsender Unterkühlung abnimmt. Die Versuchsanordnung war folgende: Die Metallstückchen wurden in Glasröhren geschmolzen und in diesen in ein Sandbad gestellt, dessen Temperatur ein wenig unter dem Schmelzpunkt der

${ }^{1}$ G. TaMm.1NN, Kristallisieren und Schmelzen. Zeitschr. phys. Chem. 25.

2 Zeitschr. physil. Chem. 23, 326. 
Metalle lag. Diese Metallstückchen zeigten grobkörnige Struktur. Metallreguli, die durch Ausgießen in Wasser oder auf eine Eisenplatte rasch gekühlt waren, zeigten feinkörnige Struktur.

Nehmen wir an, wie das schon bei anderen Körpern ${ }^{1}$ nachgewiesen ist, daB die lineare Kristallisationsgeschwindigkeit schnell mit der Unterkühlung wächst und nach Erreichen des Maximums innerhalb eines gewissen Intervalles konstant bleibt, so kann in diesem Unterkühlungsintervall die Zahl der auf der Oberflächeneinheit des Schliffes beobachteten Kristallitenquerschnitte als MaB des spontanen Kristallisationsvermögens dienen.

Da die Abküblungsgeschwindigkeit in einem weiteren Temperaturintervall nicht proportional der Differenz zwischen der Temperatur des sich abküblenden Stoffes und der des Bades ist, so erhält man auch nicht die richtige Abhängigkeit des spontanen Kristallisationsvermögens von der Ablühlungsgeschwindigkeit, sondern eigentlich nur ihren qualitativen Zusammenhang.

Die Metalle wurden in Mengen zu je $5 \mathrm{~g}$ in Jenaer Gläsern geschmolzen und darauf unter verschiedenen Bedingungen abgekühlt. An Schliffen wurde hierauf die Zahl $n$ der Kristallite auf der OberHäche $q$ (in qmm) festgestellt, wobei stets ein Mittelwert aus mehreren Zählungen genommen wurde. Bei feinkörnigen Strukturen wurden die Zählungen mikroskopisch mit Hilfe eines Okularnetzes ausgeführt.

\section{Das spontane Kristallisationsvermögen des Wismuts.}

Zur Küblung wurde zunächst in einer Reihe von Versuchen ein Paraffinbad benutzt. Das geschmolzene Metall wurde direkt ins Paraffinbad gegossen. Die Struktur der in Paraffin gekühlten Reguli war eine grobkörnige, so daB die Zahl der Strukturelemente auf der Oberflächeneinheit mit der Lupe bestimmt werden konnte. Die so gewonnenen Resultate sind aus Tabelle $1 \mathrm{zu}$ ersehen.

Tabelle 1.

$\begin{array}{rccc}t^{0} & n & \mathrm{qmm} & n / \mathrm{qmm} \\ 246 & 5 & 50 & 0.10 \\ 200 & 5.5 & 50 & 0.11 \\ 100 & 9 & 36 & 0.25 \\ 100 & 11 & 40 & 0.27 \\ 57 & 21 & 50 & 0.42 \\ 20 & 15 & 35 & 0.43\end{array}$

1 Zeitschr. physili. Chem. 25, 411. 
In einer zweiten Reihe von Versuchen werden die Wismutschmelzen in verschiedene Flüssigkeiten bei deren Siedetemperaturen gebracht. So konnte bei konstanter Badtemperatur zugleich schnelle Abkühlung erreicht werden. $\mathrm{Zu}$ diesen Versuchen diente ein kleineres Gefä $B$, das mit der Flüssigkeit gefüllt war und im Sandbade erhitzt wurde. Zur Bestimmung der Kernzahl wurden Schnitte durch den Regulus poliert und geätzt.

Tabelle 2.

\begin{tabular}{|c|c|c|c|c|}
\hline Bad & 10 & $n$ & $q \mathrm{~mm}$ & $n / q \mathrm{~mm}$ \\
\hline Anilin . & 184 & 8 & 4.5 & 2 \\
\hline Chlorbenzol & 132 & 38 & 4.5 & \multirow{2}{*}{8.2} \\
\hline, & 132 & :36 & 4.5 & \\
\hline Wasser & . 100 & 24 & 1 & \multirow{4}{*}{24} \\
\hline$"$ & . 100 & 24 & 1 & \\
\hline$n$ & 100 & 23 & 1 & \\
\hline$"$ & 100 & 26 & 1 & \\
\hline$" \quad$ & . $\quad 15$ & 22 & 1 & 22 \\
\hline $\mathrm{CO}_{2}+$ Alkohol & . 70 & 29 & 1 & \multirow{4}{*}{27} \\
\hline$"$ & . $\quad 70$ & 28 & I. & \\
\hline$"$ & 70 & 26 & 1 & \\
\hline , & 70 & 24 & 1 & \\
\hline
\end{tabular}

Bei einer dritten Reihe von Versuchen wurde das geschmolzene Wismut in eine geeignete Eisenform gegossen, welche aus zwei vollkommen aufeinander passenden Eisenplatten zusammengesetzt war. Die Eisenmasse dieser Form war groB im Vergleich zur eingegossenen Wismutmenge. Eine solche Versuchsanordnung bot zwei Vorteile: 1. sicherte die große Eisenmasse Temperaturkonstanz des Abkühlungsbades und 2. war eine schnelle Abkühlung des Regulus gewährleistet. ${ }^{1}$

Die Temperatur, bis zu welcher die Eisenplatten erwärmt wurden, lieB sich durch Auflegen von Metallstückchen von bestimmtem Schmelzpunkt (Bi, Sn) einstellen. Die Temperatur von $100^{\circ}$ wurde durch längeres Erwärmen in Wasserdampf erhalten; sehr niedrige Temperaturen konnten durch Einlegen der Eisenform in Kältemischungen erreicht werden $\left(\mathrm{CO}_{2}+\right.$ Alkohol).

Die Berührungsschicht der Reguli mit der Eisenform muB man als diejenige Stelle ansehen, in welcher sich zuerst die Kristallzentren bilden. Von hier aus dringt die Kristallisation nach innen

1 Absolute Wärmeleitfähigkeit: $k_{\text {Eisen }}=0.1240, k_{\mathrm{Bi}}=0.0192$ (LandoltTabellen). 
vor, den Wärmeabflußlinien folgend. Die Richtung dieser WärmeabfluBlinien kann man an der Richtung der Kristalle erkennen: dieselben zeigen nämlich eine in der Richtung senkrecht zur Abkühlungsfläche verlängerte Form. Unter den angegebenen Versuchsbedingungen war infolge des größeren Wärmeleitvermögens des Eisens die Abkühlungsgeschwindigkeit an der Oberfläche des Regulus gröBer als in seinen inneren Schichten, daher wurde zur Zählung der Kristalliten stets die Basis der Reguli schwach angeschliffen.

\begin{tabular}{rrcc}
\multicolumn{4}{c}{ Tabelie 3.} \\
$t^{0}$ & $n$ & qmm & $n /$ qmm \\
260 & 15 & 78 & 0.2 \\
220 & 11 & 78 & 0.14 \\
100 & 6 & 0.33 & 18 \\
20 & 16 & 0.07 & 228 \\
-70 & 20 & 0.07 & 286
\end{tabular}

In der Eisenform war die Abkühlungsgeschwindigkeit offenbar größer als in den siedenden Flüssigkeiten, da das Gießen in die Eisenform ein sehr viel kleineres Korn des Wismuts ergab als das Gießen in die auf ihren Siedepunkt erhitzten Flüssigkeiten.

\section{Das spontane Kristallisationsvermögen des Antimons.}

Hier wurde in einer Reihe von Versuchen zunächst derart verfahren, daß das geschmolzene Metall in einen Fingerhut gegossen wurde, welcher im Sandbade stand und auf verschiedene Temperaturen erhitzt war. Die Reguli zeigten großkörnige Struktur, da das geringe Wärneleitungsvermögen des Sandes eine langsame Abkühlung bedingte. Der EinfluB der Badtemperatur lieB sich auch hier verfolgen, wie Tabelle 4 zeigt.

\begin{tabular}{rrcc}
\multicolumn{4}{c}{ Tabelle 4.} \\
$t^{0}$ & $n$ & qmm & $n / \mathrm{qmm}$ \\
560 & 18 & 4.5 & 4 \\
400 & 38 & 4.5 & 8.4 \\
200 & 57 & 4.5 & 13
\end{tabular}

Bei einer weiteren Versuchsreihe wurde das Antimon nach dem Schmelzen in eine eiserne Form gegossen, und in der Weise untersucht, wie das schon bei Wismut beschrieben ist.

Das spontane Kristallisationsvermöæen wächst also anfangs langsam mit der Abkühlungsgeschwindigkeit, steigt dann schnell bis zu einem Maximum an, um weiter innerhalb eines gewissen Intervalles 
182 E. Belier. Das spont. Kristallisationsvermägen bei Wismut u. Antimon.

von der Temperatur unabhängig zu werden, und schließlich bei sehr tiefer Badtemperatur abzunehmen.

\begin{tabular}{rccc}
\multicolumn{4}{c}{ Tabelle 5.} \\
$t^{0}$ & $n$ & qmm & $n /$ qmm \\
600 & 12 & 56 & 0.21 \\
500 & 15 & 75 & 0.20 \\
400 & 73 & 1 & 73 \\
200 & 80 & 1 & 80 \\
20 & 63 & 1 & 63 \\
-70 & 29 & 4.5 & 6.4
\end{tabular}

Die Reguli, welche bei Temperaturen höher als $500^{\circ}$ abgekühlt wurden, zeigten durchweg grobkörnige Strukturen, die bei $400^{\circ}$ und bei $200^{\circ}$ gekühlten, eine feinkörnige mit ungefähr gleicher Anzahl der Kristallindividuen. Die bei Zimmertemperatur $\left(20^{\circ}\right)$ gekühlten Reguli zeigten ebenfalls eine feinkörnige Struktur, jedoch mit etwas größeren Kristallen, als im vorhergehenden Falle, und die bei $-70^{\circ}$ gekühlten Reguli waren erheblich grobkörniger als diese. GieBt man das flüssige $\mathrm{Sb}$ direkt in die Kältemischung $\left[\mathrm{CO}_{2}\right.$-Alkohol], so erhält man für $n / q m m$ im Mittel ebenfalls die Zahl 6 .

Da bei genügend großer Abkühlungsgeschwindigkeit das spontane Kristallisationsvermögen des $\mathrm{Sb}$, welches der Zahl n/qmm proportional ist, abnimmt, so wäre es vielleicht möglich, bei Anwendung sehr starker Unterkühlung das Antimon im amorphen Zustande zu erhalten, also wahrscheinlich als schwarzes Antimon' in der Form, wie A. STock und W. SIEBERT ${ }^{1}$ das Antimon durch Kondensation seines Dampies oder durch lange Oxydation von Antimonwasserstoff bei $-40^{\circ}$ dargestellt haben, und von dem sie wahrscheinlich gemacht haben, daß es wirklich amorph ist.

${ }^{1}$ Ber. deutsch. chem. Ges. :3S, 3839.

Göttingen, Institut für physilkalische Chemie.

Bei der Redaktion eingegangen am 28. August 1912. 